\title{
BACK TO BENTHAM? EXPLORATIONS OF EXPERIENCED UTILITY*
}

\author{
Daniel Kahneman \\ Peter P. WAKKer \\ RAKESH SARIN
}

Two core meanings of "utility" are distinguished. "Decision utility" is the weight of an outcome in a decision. "Experienced utility" is hedonic quality, as in Bentham's usage. Experienced utility can be reported in real time (instant utility), or in retrospective evaluations of past episodes (remembered utility). Psychological research has documented systematic errors in retrospective evaluations, which can induce a preference for dominated options. We propose a formal normative theory of the total experienced utility of temporally extended outcomes. Measuring the experienced utility of outcomes permits tests of utility maximization and opens other lines of empirical research.

\section{INTRODUCTION}

The concept of utility has carried two quite different meanings in its long history. As Bentham [1789] used it, utility refers to pleasure and pain, the "sovereign masters" that "point out what we ought to do, as well as determine what we shall do." This usage was retained in the economic writings of the nineteenth century, but it was gradually replaced by a different interpretation [Stigler 1950]. In current economics and in decision theory, the utility of outcomes and attributes refers to their weight in decisions: utility is inferred from observed choices and is in turn used to explain these choices. To distinguish the two notions, we shall refer to Bentham's concept as experienced utility and to the modern usage as decision utility. With few exceptions, ${ }^{1}$ experienced utility is essentially ignored in modern economic discourse. The rejection of experienced utility is justified by two standard arguments: (i) subjective hedonic experience cannot be observed or measured; (ii) choices provide all necessary information about the utility of outcomes because rational agents who wish to do so will optimize their hedonic experience. Contrary to this position,

*This work is dedicated to the memory of Amos Tversky. We are grateful to many friends and colleagues who commented on earlier versions. Special thanks are due to Peter Diamond and David Laibson. The usual caveats apply.

1. For some recent attempts to use subjective measures in economic analyses, see Clark and Oswald [1994], Kapteyn [1994], Tinbergen [1991], and van Praag [1991].

(C) 1997 by the President and Fellows of Harvard College and the Massachusetts Institute of Technology.

The Quarterly Journal of Economics, May 1997. 
we will argue that experienced utility is both measurable and empirically distinct from decision utility.

The following example illustrates the distinction between the two concepts of utility. ${ }^{2}$ A patient suffering from unusually profound amnesia has two toasters in his kitchen. The toaster on the right functions normally. The toaster on the left delivers an electric shock when the toast is removed. The patient's gasp and quick retraction of his hand indicate that the shock is painful. Because the patient does not remember the experience, however, he does not anticipate the shock the next morning, and is consequently indifferent between the toasters. The patient's decision utility for using the two toasters is equal, but his experienced utilities are quite different. The patient's choices of the left-hand toaster will not maximize utility in Bentham's sense. As we will show later, discrepancies between decision utility and experienced utility are not restricted to such pathological cases. Systematic errors in the evaluation of past events and decisions that do not maximize future experienced utility can be observed in decision makers whose cognitive functions are normal. These observations raise doubts about a methodology in which observed choices provide the only measure of the utility of outcomes.

Pleasure and displeasure are attributes of each moment of experience, but the outcomes that people value are normally extended over time. The basic building block of experienced utility in our analysis is instant utility: a measure of hedonic and affective experience, which can be derived from immediate reports of current subjective experience or from physiological indices. Instant utility corresponds to the dimension of "intensity" in the writings of Bentham, Jevons, and Edgeworth. The focus of our analysis is the evaluation of temporally extended outcomes (TEOs), such as a single medical procedure or the concatenation of a Kenya safari and subsequent episodes of slide-showing and storytelling. Two measures of the experienced utility of temporally extended outcomes will be considered. Remembered utility is a measure on past TEOs, which is inferred from a subject's retrospective reports of the total pleasure or displeasure associated with past outcomes. Total utility is a normative concept. It is a measure on possible TEOs, which is constructed from temporal profiles of instant utility according to a set of normative rules. Decision utility is a measure on TEOs which is inferred from choices, either by direct comparisons of similar objects or by indi-

2. This example was suggested by Paul Romer. 
rect methods, such as elicited willingness to pay. Finally, we will discuss predicted utility, which refers to beliefs about the experienced utility of outcomes. The relations among the various utility concepts define a complex agenda for research. Figure I lists some of the questions that arise. Several of these questions will be addressed here.

Section I discusses the adaptive significance and the measurement of instant and remembered utility. Section II describes studies in which participants provided a continuous record of instant utility during an episode (e.g., a short film, or a medical procedure), and later reported a global evaluation of the episode (remembered utility), or made choices about which of several episodes to repeat (decision utility). These studies are concerned with the determinants of remembered utility (question \#4 in Figure I) and with the role of remembered utility in choice (\#8). The duration of episodes played very little role in subjects' retrospective evaluations in these experiments, contrary to an obvious normative rule (\#5). Subjects also made choices that exposed them to avoidable pain or discomfort, in violation of dominance. Their decisions did not maximize experienced utility (\#9), although they may have maximized remembered utility (\#10).

Section III and the Appendix present a normative theory of total utility, which yields rules for the evaluation of temporally extended outcomes on the basis of a temporal profile of instant utility (\#3). The theory assumes time-neutral weighting of instants (no time preference) and derives temporal integration as the principle of global evaluation. Section IV elaborates on the consequences of accepting experienced utility as a measure of the quality of outcomes, distinct from decision utility. The section also includes a brief review of some results concerning the accuracy of predicted utility (\#6). Section V concludes. Proofs are presented in the Appendix.

An important reservation should be stated early. Our normative treatment of the utility of temporally extended outcomes adopts a hedonic interpretation of utility, but no endorsement of Bentham's view of pleasure and pain as sovereign masters of human action is intended. Our analysis applies to situations in which a separate value judgment designates experienced utility as a relevant criterion for evaluating outcomes. ${ }^{3}$ This set does not include all human outcomes, but it is certainly not empty. [1993], Nussbaum and Sen [1993], and Sen [1991]. 


\section{Stream of Outcomes $\stackrel{1,2}{\longrightarrow}$ Instant Utility}

1) What utility functions relate hedonic experience to outcomes?

2) What are the dynamics of experienced utility?

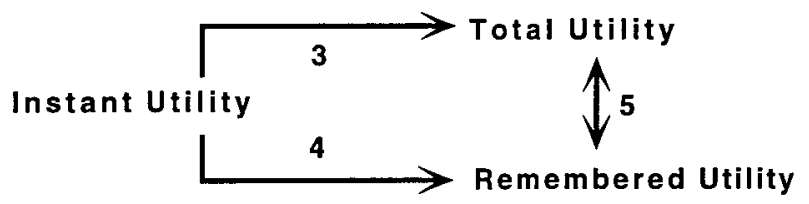

3) What are the rules of evaluation for extended outcomes?

4) How is the remembered utility of extended outcomes determined?

5) Does remembered utility conform to normative rules?

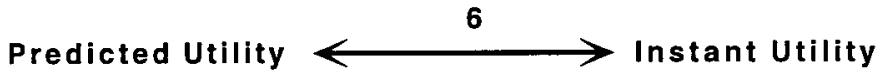

6) Do people accurately forecast the instant utility of outcomes?

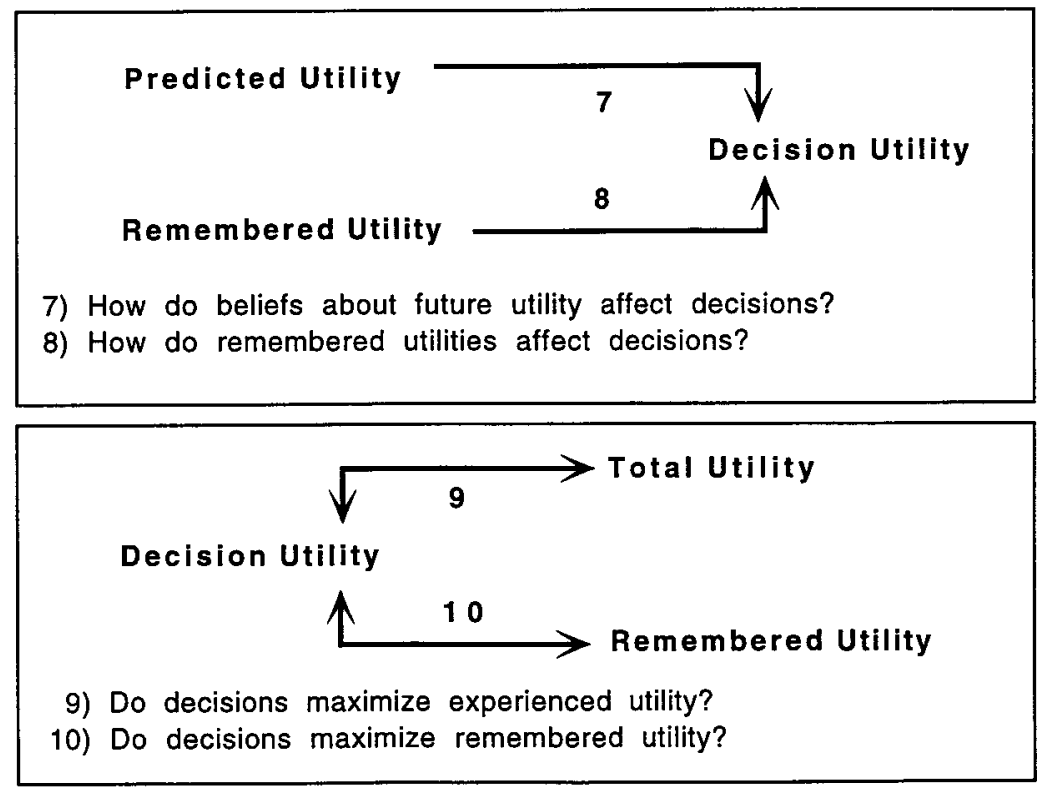

Figure I

Research Designs for the Study of Experienced Utility 


\section{The Functions of Experienced Utility}

We have distinguished two descriptive notions of experienced utility: instant utility is the pleasure or distress of the moment; remembered utility is the retrospective evaluation of a temporally extended outcome. This section presents a brief introduction to the biology and psychology of these forms of utility, and to some issues of measurement.

The adaptive functions of pleasure and pain can be deduced from the conditions under which these experiences are biologically programmed to occur. Pleasure is evidently a "go" signal, which guides the organism to continue important activities such as foreplay or consuming sweet, energy-rich food. Pain is a "stop" signal, which interrupts activities that are causing harm, such as placing weight on a wounded foot. The common characteristic of the basic forms of pleasure and distress is that they regulate the response to the current situation.

To achieve its function in the control of immediate consumption or escape, hedonic value must accurately reflect the needs of the moment. The palatability of salt, for example, increases in conditions of sodium depletion. On a different time scale, the hedonic value of food changes substantially during a single feeding episode, and normally drops to zero or becomes negative when feeding continues beyond satiation [Cabanac 1971]. Of course, not all human pleasures and pains are biologically programmed in detail. Prior consumption experiences and various cultural and social influences can alter the hedonic value of stimuli, as when people learn to like coffee or chili peppers, develop a dislike for rich desserts, or acquire a passion for opera. Furthermore, positive or negative instant utility can be evoked by social stimuli, such as smiles or frowns, and by purely internal events such as memories of embarrassment or amusing thoughts [Schelling 1984]. In spite of the immense diversity in the occasions that evoke pleasure (or displeasure) in the human adult, the hedonic attribute that they share is salient and readily recognized.

The view that hedonic states cannot be measured because they are private events is widely held but incorrect. The measurement of subjective experiences and the determination of the functions that relate subjective variables to features of present and past stimuli are topics in the well-established field of psychophysical research [Stevens 1975; Wegener 1982]. The loudness of a noise and the felt temperature of a limb are no less subjective 
than pleasure and pain. The main argument for considering these experiences measurable is that the functions that relate subjective intensity to physical variables are qualitatively similar for different people. For example, reported subjective intensity is often a power function of physical magnitude, with an exponent that varies for different sensory dimensions. Pleasure and distress have the same status: the psychophysical functions that govern the pleasure of drinking sugar solutions and the pain of electric shock are orderly and interpersonally similar [Stevens 1975]. Verbal or numerical reports of hedonic value can be supplemented by physiological indicators of emotional quality and intensity, including objective measurements of subtle facial expressions [Frank 1988]. Although the correlations among these measures are imperfect, the variance they share can serve to operationalize the concept of instant experienced utility.

As an object of measurement, experienced utility has much in common with subjective temperature. Like hot and cold, the experiences of pleasure and distress differ in quality. A scale that ranges from extreme pleasure to extreme distress (or from very hot to very cold) effectively comprises separate scales for two distinct attributes. The two scales are joined by a distinctive neutral point: "neither hot nor cold," "neither pleasant nor unpleasant." The stimulus that gives rise to a neutral experience may be different in different contexts, but the neutral experience itself is constant. A standard demonstration involves two pails of warm water, one warmer than the other. A subject who immerses one hand in each of these pails will eventually report that both hands feel alike, neither hot nor cold. The neutral value provides a natural zero point for bipolar dimensions. Because of its distinctiveness, the neutral value can be used with some confidence to match thermal (or hedonic) experiences across time for a given individual, and even to support interpersonal comparisons [Kahneman and Varey 1991].

Remembered utilities also have an adaptive function: they determine whether a situation experienced in the past should now be approached or avoided. Unlike pain and pleasure, which control behavior in the current situation, learned attractions and aversions adjust current behavior to the remembered evaluations of events in the past. Remembered utilities can be measured in humans by reported evaluations of past experiences, or by physiological indications of the emotion aroused by reminders of 
the event. Remembered utilities can also be inferred from the approach or avoidance tendencies that they induce.

Like the hedonic system, the system that governs the remembered evaluation of past experiences has been under evolutionary pressure. However, the effect of natural selection is to increase overall fitness, not necessarily to produce organisms that maximize pleasure and minimize pain over time. For example, the fear displayed by rats that have been repeatedly exposed to an electric shock is affected by the intensity of the shock, but not by its duration [Mowrer and Solomon 1954]. The next section reports similar observations of duration neglect in human subjects. These findings suggest that the system that forms and stores evaluations of situations is not designed to optimize experienced utility.

\section{The Experienced Utility and Decision Utility of Episodes}

The present section summarizes the results of a series of studies of remembered utility, which investigated two questions: (i) what attributes of the temporal profile of instant utility determine the remembered utility of an episode? (ii) are biases in remembered utility reflected in subsequent choices? A central issue in these studies was the role of duration as a determinant of the utility of episodes. The results support two generalizations. (1) Peak-End evaluation: the remembered utility of pleasant or unpleasant episodes is accurately predicted by averaging the Peak (most intense value) of instant utility (or disutility) recorded during an episode and the instant utility recorded near the end of the experience. (2) Decisions by remembered utility: when given the choice of which of several episodes to repeat, individuals generally choose the episode that has the highest remembered utility. Two consequences of Peak-End evaluation have been confirmed, both in measures of remembered utility and in choices. (1) Duration neglect: the duration of experiences has little or no independent effect on their remembered utility. (2) Violations of temporal monotonicity: the remembered disutility of an aversive episode can be reduced by adding an extra period of discomfort that reduces the Peak-End average.

Several experimental designs were employed in this research. The participants in the experiments generally provided continuous or intermittent measures of instant utility, which per- 
mitted a computation of the Peak-End average. The duration of the episodes was allowed to vary naturally in some studies, and was varied experimentally in others. Diverse measures of remembered utility were used, including ratings of overall aversiveness and comparative evaluations. Choices were recorded in some of the experiments.

Participants in experiments reported by Fredrickson and Kahneman [1993] were exposed to sixteen short, plotless film clips. Half of these films were pleasant (e.g., views of a coral reef), half were aversive (e.g., an amputation). There were two versions of each film: a short version (approximately 45 seconds) and a longer version (approximately 120 seconds), with no notable variation of content or affective value. Each subject saw the short versions of half the films and the long versions of the others. The subjects used a sliding knob that controlled an array of colored lights to provide a continuous measure of the instant utility (or disutility) they experienced during the presentation of each film [Gottman and Levenson 1985].

Several measures of remembered utility were used. Participants in one study answered the question "Overall, how much pleasure [displeasure or discomfort] did you experience during the film?" Because this instruction could perhaps be interpreted as referring to the most extreme affect experienced during the episode, another measure was used in a subsequent study. Participants were told that the researchers sought their advice to compile videotapes that subjects would view in future experiments. The task was to rank the films according to the contribution each made to the overall affective experience of viewing the videotape. Pleasant and unpleasant clips were ranked separately. The ranking of the aversive clips was explained as follows: "The unpleasant videotape will consist of several of the unrelated unpleasant clips that you just saw. Of course people who will see this unpleasant videotape will experience some unpleasantness. . . Y Your task is to help us select the unpleasant clips we should include in order to MINIMIZE the overall experience of unpleasantness for most people."

The results of both experiments generally supported the hypotheses of Peak-End evaluation and duration neglect. In a within-subject regression analysis of the first experiment, the mean correlations between the Peak-End average of the real-time measure and ratings of remembered utility were .77 for pleasant films and .69 for aversive films. When the Peak-End measure was 
statistically controlled, the mean correlations between the remembered utility of films clips and their duration were .06 and -.02 , respectively. ${ }^{4}$ Very similar results were obtained with the ranking measure used in the second experiment. The subjects' rankings of the film clips they wished to include in a videotape were entirely unaffected by duration.

Duration neglect and Peak-End evaluation were confirmed in a more realistic setting, using a between-subjects design [Redelmeier and Kahneman 1996a]. The participants were patients undergoing a colonoscopy in a Toronto hospital. The patients reported their level of discomfort every 60 seconds throughout the procedure (see Figure II). Later, the patients reported the remembered disutility of the procedure, using several different scales. The patients rated the "total amount of pain experienced" on a 10-point scale. They also ranked a list of unpleasant experiences, which included "the colonoscopy you have just had" along with other incidents, such as stubbing a toe and "an average visit to the dentist." The patients were also required to indicate a preference between a repeat colonoscopy and a barium enema. PeakEnd evaluation and duration neglect were observed for all these measures. For example, the correlation of the Peak-End average with the global rating of the procedure was .67. Although there was substantial variability in the duration of the colonoscopies that different patients experienced (the mean duration was 23 minutes, with a standard deviation of 13 minutes), the correlation between duration and the global evaluation of the procedure was only .03. Figure II shows illustrative data for two patients. Although most observers judge that Patient B had a worse experience, the Peak-End rule correctly predicts a worse retrospective evaluation by Patient A, whose End rating was much higher.

A notable feature of this study is that robust results were obtained in a design which effectively assumes the interpersonal comparability of measures of experienced utility. Several findings support the conclusion that people share a common scale for both instant and remembered utility. First, the requirement to provide ratings of discomfort during the procedure was eliminated for a group of 53 patients; an observer who attended the examination

4. The first-order correlation between disutility and duration was significant for the aversive clips, but this correlation was produced by a general tendency for the ratings of instant discomfort to increase during the exposure of these clips. A pattern of escalating discomfort is common in aversive situations, where it induces a positive correlation between duration and the Peak-End measure. 

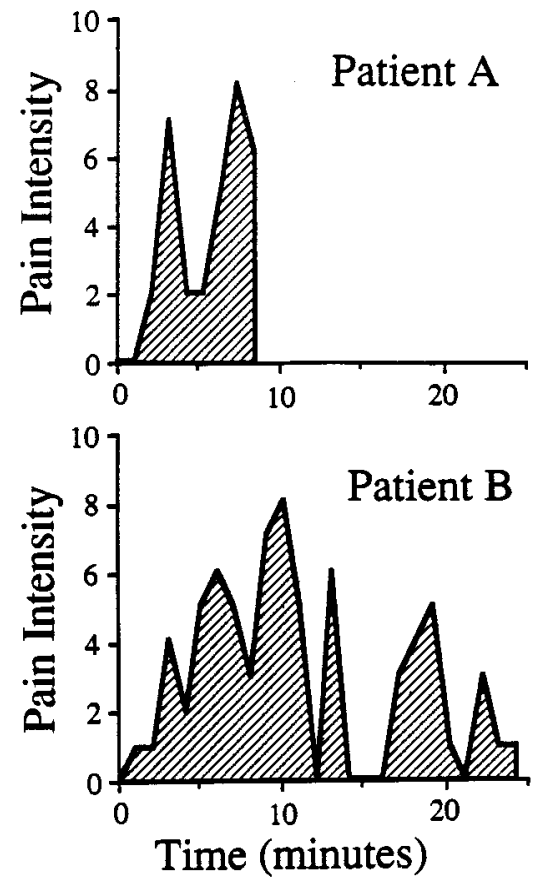

FIgURE II

Pain Intensity Reported by Two Colonoscopy Patients (from Redelmeier and Kahneman [1996]).

estimated the patient's discomfort every 60 seconds. The PeakEnd average of the observer's estimates predicted the retrospective evaluations subsequently elicited from patients $(r=.71)$. Second, the physicians who administered the procedure also provided several ratings of each patient's overall discomfort. The correlation between the ratings of patients and physicians was .67. The physicians evidently applied the same model of evaluation (Peak-End rule and duration neglect) as did the patients. One of the questions put to the physicians was whether more anesthetic should have been used. The correlation between answers to this question and the duration of the procedure was .05.

The psychological interpretation of this pattern of findings is straightforward. The subjects and observers in these studies apparently constructed a representative moment of each episode they had experienced (or observed), and used this representative moment as a proxy to evaluate the utility of the entire episode. 
The representative moment is better described as a collage than as a precise memory of a particular instant of the experience; it is affected by several singular instants, including the peak and the end of the episode. The duration of the episode is not included in this basic representation, even when it is accurately known [Fredrickson and Kahneman 1993; Kahneman, Fredrickson, Schreiber, and Redelmeier 1993; Varey and Kahneman 1992]. The Peak-End representation controls an emotional response, which is reflected in the global evaluations of the episode, and which we label remembered utility. As was previously observed in the fear response of lower animals [Mowrer and Salomon 1954], this emotional response is essentially independent of duration. When their attention is directed to it, of course, human subjects universally agree that duration is a relevant attribute of pleasant or aversive episodes.

The Peak-End rule entails violations of temporal monotonicity: adding an extra period of discomfort to an aversive episode will improve the remembered utility of the episode if the added period ends on a less unpleasant note. Redelmeier and Kahneman [1996b] tested this prediction in a clinical setting, with a large population $(N=682)$ of patients undergoing a colonoscopy. Half the patients were randomly assigned to a condition in which the procedure was extended by leaving the colonoscope in place for about a minute after the completion of the clinical examination. The patients were not informed of the experimental treatment. The experience during the added period was mildly uncomfortable (certainly less preferred than the alternative of removing the instrument) but for many patients less painful than the preceding moments. As predicted by the Peak-End rule, the prolongation of the colonoscopy yielded a highly significant improvement in the global evaluations of the procedure. The purpose of the experiment was to test the hypothesis that an intervention that reduces the remembered disutility of the procedure could increase patients' willingness to undergo further colonoscopies if needed.

We next consider whether the biases of remembered utility transfer to decision utility. As we saw earlier, the description of behavior as governed by pleasure and pain is only applicable to consumption and escape. In many other situations the sovereign masters that determine what people do are not pleasure and pain, but memories of pleasure and pain. Indeed, the only utility that people (and other organisms) can learn from personal experi- 
ence to maximize is the utility that they remember. If a retrospective evaluation distorts the hedonic quality of an experience, subsequent preferences will be governed by the biased evaluation, not by the original experience. This reasoning implies that the violations of temporal monotonicity observed in ratings of remembered utility should also be observed in actual choices. Several experiments have tested this prediction.

Participants in an experiment reported by Kahneman et al. [1993] were advised that they would undergo painful experiences, and as part of the informed consent procedure were asked to immerse a hand to the wrist in very cold water, for ten seconds. They were told to expect three trials of this kind, but only two were actually conducted. In the Short trial the subject kept one hand in water at $14^{\circ} \mathrm{C}$ for 60 seconds, after which he was allowed to remove the hand from the water and to dry it with a warm towel. In the Long trial the immersion lasted a total of $90 \mathrm{sec}-$ onds. Water temperature was kept at $14^{\circ}$ for the first 60 seconds, at which point (unbeknownst to the subject) the experimenter caused the temperature of the water to rise gradually from $14^{\circ}$ to $15^{\circ}$ over the next 30 seconds. Different hands were used for the Short and for the Long trials. Half the subjects experienced the Short trial before the Long one; the sequence was reversed for the other subjects. The trials were separated by seven minutes during which the subject performed an unrelated task, which was resumed after the second trial. Seven minutes later, the subject was called in for a third trial, informed that one of the two previous procedures would be repeated exactly, given a choice of whether the first or the second trial should be repeated, and asked to answer several questions about the first two trials.

Several experiments were conducted in this general design, with minor variations of procedure. The 32 participants in the main experiment used a "discomfort meter" to provide a continuous indication of the pain they experienced, on a scale ranging from 0 (no pain at all) to 14 (intolerable pain). The real-time records permitted a classification of subjects into two groups. Most subjects indicated a decline of two or more points on the pain scale during the last 30 seconds of the experiment; the mean level of pain that these subjects recorded was 8.5 at 60 seconds and 4.4 at 90 seconds. For these subjects the Peak-End average was lower on the Long than on the Short trial. As expected, a large majority of them (17 of 21) preferred to repeat the Long trial. The other eleven subjects did not indicate a significant decrease of 
pain as the temperature of the water was raised. For these subjects the Peak-End average was very similar on the Short and on the Long trial. Only five of the eleven subjects preferred the Long trial. The difference between the preferences of the two groups was statistically significant, as was the overall preference for the Long trial (69 percent for the 32 subjects). To check the robustness of the results, the experiment was replicated with 37 new subjects, without the requirement to report pain in real time. The results confirmed the original findings: 65 percent of the new subjects preferred to repeat the Long rather than the Short trial.

Subjects did not spend much time in analytic comparisons before choosing which trial to repeat: they simply determined which of the two memories they disliked less. Postexperimental interviews and other observations provided no support for more speculative accounts. First, the interviews confirmed that the Long trial was not chosen out of a wish to endure more pain. Indeed, all subjects quickly removed their hand from the water as soon as they were allowed to do so. Second, the subjects never described the last segment of the Long trial as a pleasurable experience. Third, they did not prefer the Long trial because it yielded a more pleasant postimmersion experience. On the contrary, pleasurable relief was more intense after the Short trial.

The neglect of duration in this experiment represents neither a failure of duration memory (most subjects could correctly answer a question about the durations of the two trials) nor a deliberate strategy. We have observed in unpublished experiments that subjects who are are asked to choose between hypothetical profiles of a Long and Short trial almost invariably obey dominance, although most subjects follow the Peak-End rule and implicitly violate dominance when judging a series of hypothetical profiles one by one [Varey and Kahneman 1992]. In the coldwater experiment, the choice is made by comparing remembered utilities, without performing the explicit comparison that could reveal the Long trial to be a dominated option.

The violations of dominance observed in the cold-water experiment have been replicated with loud sounds as aversive stimuli [Schreiber and Kahneman 1996]. Subjects heard pairs of sounds in immediate succession, then indicated which of the two sounds they would rather hear repeated in a subsequent phase of the experiment. Some of the stimuli were constructed along the lines of the cold-water experiment. For example, one of the pairs of stimuli that the subjects heard was (i) ten seconds of an 
unpleasant sound at $78 \mathrm{db}$, and (ii) the same sound followed by four additional seconds at a lower intensity (66db). There was a significant preference for repeating the Long sounds in such pairs. The pattern did not change in the course of a series of ten decisions, including five which pitted a Long sound against a Short one.

The experiments reviewed in this section all involved short episodes, in which the subjects were passive. The episodes were also hedonically homogeneous; none combined pleasant and unpleasant moments. Although the exact pattern of findings may be specific to this particular class of situations, the results support two general observations. The first observation is methodological: some peculiarities of preferences are unlikely to be understood, or even detected, without first understanding experienced and remembered utility. The second observation is that the statement that decisions maximize utility is not a tautology; it can be proved false.

\section{Normative Analysis}

The subjects in the experiments described in the preceding section violated a compelling principle of temporal monotonicity. We therefore claimed to have observed preferences that do not maximize experienced utility, but we did not specify precisely what maximization means in the context of temporally extended outcomes. We turn to this task now.

The measured primitive of experienced utility is instant utility, the hedonic value of a moment of experience as immediately reported or recorded. Significant outcomes last more than an instant, however, and the total utility of such outcomes must therefore be derived from a temporal profile of instant utility. The theory presented in this section specifies the conditions under which the total utility of an extended outcome is the temporal integral of some transformation of instant utility. This normative analysis serves two goals. On the one hand, it identifies axioms of total utility that yield a rule of temporal integration and allows an evaluation of the intuitive appeal of these axioms. On the other hand, the analysis constrains the measure of instant utility, which must provide all the information required for an adequate assessment of total utility.

The normative treatment of total utility can be interpreted in at least two ways. The most straightforward interpretation is 
that retrospective evaluation of outcomes is a cognitive activity in which people routinely engage, much as they engage in grammatical speech or in deductive reasoning. Normative standards apply to each of these activities. Rules and errors can be identified for grammar, for deduction, and also for retrospective evaluation. Economists who associate normative analysis exclusively with decisions may prefer another interpretation, in which total experienced utility is the objective function that a benevolent social planner would wish to maximize.

Temporal integration is an obvious candidate for a normative principle of total utility. A sketch of the idea can be found in Edgeworth [1881, p. 101]: “. . . let there be granted to the science of pleasure what is granted to the science of energy; to imagine an ideally perfect instrument, a psychophysical machine, continually registering the height of pleasure experienced by an individual. ... The continually indicated height is registered by photographic or other frictionless apparatus upon a uniformly moving vertical plane. Then the quantity of happiness between two epochs is represented by the area contained between the zero-line, perpendiculars thereto at the points corresponding to the epochs, and the curve traced by the index."

The analysis that follows assumes ordinal measurement of both instant utility and total utility. Stronger results that require cardinal measurement are also mentioned. Ordinal measurement of instant utility implies that any two moments of experience can be compared, to establish which of them carries the higher hedonic value. The measure of instant utility should also permit reliable identification of the hedonically neutral state, which plays a special role in the analysis. As was mentioned earlier, these requirements are not beyond the realm of the possible.

The following terms will be used; formal definitions are presented in the Appendix. An episode is a connected time interval described by its temporal coordinates. A group of one or more temporally disjoint episodes is called a temporally extended outcome (TEO). The utility profile of a TEO assigns a level of instant utility to each time point (see Figure II; note that the ordinate is inverted in this figure, to correspond to the format of the patients' ratings).

Utility profiles can be concatenated. If $f$ is a utility profile of duration $x$ and $g$ is a utility profile of duration $y$, then their concatenation is of duration $x+y$, coinciding with $f$ on the first part and with $g$ on the second. For example, imagine that the two 
disutility profiles shown in Figure II were produced by the same individual, during two colonoscopies conducted on separate days. The patient's total experience of colonoscopy could be represented by concatenating the two profiles. The analysis assumes that the calibration of the scale of instant utility is constant, both for the episodes included in a TEO and for TEOs that are compared with each other. Of course, this requirement is likely to be violated when the situations are radically different, as in comparisons of the experiences of a person as a baby and as an adult.

We now present axioms for total utility that characterize the integration rule. The first axiom considers neutral utility profiles, defined as profiles in which instant utilities are hedonically neutral, i.e., "neither good nor bad." It plays an essential role in comparisons of TEOs that differ in duration.

Axıom 1 [concatenation of neutral utility profiles]. The total util-

ity of a utility profile is not affected by concatenation with a neutral utility profile.

The conditions that we impose next reflect the consequentialist requirement that a measure of instant utility should comprise all the information required for the determination of total utilities.

Axıom 2 [monotonicity in instant utility]. Increases of instant utility do not decrease the total utility of a utility profile.

The axiom implies that any local improvement of instant utility is perceived as an improvement of total utility, irrespective of earlier or subsequent instant utilities.

Axıom 3 [monotonicity in total utility]. In a concatenation of two utility profiles, replacing one profile by another with a higher total utility will increase the total utility of the concatenation.

Axiom 3 implies separability of disjoint time periods. If one utility profile is better than another, then this is not affected by the utility profiles that precede and follow them. The instant utility ratings must be "sufficient statistics" in the sense that all the information needed to evaluate the goodness of an episode must be incorporated in its utility profile. In particular, any effects of previous or anticipated consumption on the utility of present consumption must be incorporated in the measure of instant utility. Separability is commonly used to characterize additive decompos- 
ability over disjoint time intervals; thus, Axiom 3 is the crucial axiom for the integral representations obtained in this section.

Theorem 1 . The three axioms above hold if and only if there exists a nondecreasing ("value") transformation function of instant utility, assigning value 0 to 0 , such that total utility orders utility profiles according to the integral of the value of instant utility over time. ${ }^{5}$

The normative determination of total utility consists of two parts. Instant utilities are first measured and then transformed by means of the value function. As noted earlier, the same normative analysis applies to the subject who reported the instant utilities and to an observer with access to the utility profile. The theorem assumes that the instant utility ratings are ordinal except for the zero point. The transformation yields a ratio scale, which is unique up to multiplication by a positive scale factor. The present formulation and axioms differ from other treatments in the time preference literature mainly in our use of the concatenation operation. This operation is applicable because global evaluations are undated. Our derivation combines techniques from "Höelder's theorem" with Gorman's [1968] result on the representation of separable preferences.

Separability in terms of outcomes is not reasonable, and counterexamples are easily constructed. For example, the relative values of a strenuous walk and of reading a book in the afternoon depend on whether the individual spent the morning playing tennis or working at a computer. One response to the problem is a nonadditive model of preferences over outcome profiles that permits violations of separability, as in Gilboa [1989] and Constantinides [1990]. Our model retains separability because it assumes that utility profiles are specified in terms of instant utilities, not outcomes. Using measurements of instant utility, we would expect to find that the relative instant utilities of alternative programs for the afternoon depend strongly on how the morning was spent. Separability is reasonable when outcomes are described in terms of instant utility, provided that instant utility incorporates all order effects and interactions between outcomes. This treatment is similar to Stigler and Becker

5. Because the term utility is already used in another sense, we resort to the term "value" to designate the transformation of instant utility. 
[1977], who achieved separability by redefining outcomes as appropriate conjunctions of present and past consumption.

An implication of Theorem 1 is that the ordering of time points in a utility profile does not affect total utility.

Corollary 1. The total utility of a utility profile, and hence of a TEO, depends only on the generated time distribution over instant utility, i.e., the total time spent at each region of instant utility.

This corollary reflects the time neutrality of the model.

The analysis becomes simpler if cardinal measurement of instant utility can be assumed, so that differences of instant utility are meaningful. Transformation by the value function is not required in this case. The following axiom characterizes cardinal measurability of instant utility.

Axiom 4 [cardinality of instant utility]. The ordering of total utility of two utility profiles does not change if for both the instant utility level is increased by the same constant over an equally long time period.

Theorem 2. Axioms 1, 2, and 4 hold if and only if total utility orders utility profiles according to the integral of instant utility over time.

Theorems 1 and 2 can be simplified further if total utility is measured at a cardinal level (Theorem A.2 and Corollary A.1 in the Appendix).

Finally, we note the following simple implication, which is satisfied in each of the models, and was tested empirically in the experiments described in the preceding section.

Corollary 2 [time monotonicity]. Axioms 1 (concatenation of neutral utility episodes) and 2 (monotonicity of instant utility) imply that concatenation with a utility profile that lies entirely above zero does not lower total utility, and concatenation with a wholly negative profile does not raise total utility.

\section{Discussion}

The object of this paper was to show that experienced utility is governed by a distinctive normative logic, that it is potentially measurable, and that the empirical study of experienced utility 
is relevant to some topics of concern to economists. We discuss these issues in turn.

1. Normative issues. Our analysis of total utility assumes a time-neutral perspective and emphasizes duration as a feature of outcomes. Neither idea is new. There is a long history of debate about the normative status of time preferences, especially about the rationality of discounting the future [Loewenstein 1992], and duration is represented in any treatment of utility over time. However, the context of experienced utility highlights particular aspects of these ideas.

Although many authors have argued for a time-neutral perspective, the prevailing position in the economic literature is that utility discounting is compatible with rationality. The exclusive focus on decisions in economic thinking helps explain the tolerance for discounting: decision utility is evaluated from the perspective of an agent for whom immediate outcomes indeed loom larger than more distant ones. In contrast, the total experienced utility of an outcome is not evaluated from any particular point in time. The entire utility profile of the outcome must be known, but the date of the evaluation is irrelevant. The same reasoning extends naturally to time-neutral weighting of early and late segments of extended outcomes. Although other models of total utility could be formulated, time neutrality appears most appropriate for the evaluation of experienced utility. The adoption of this principle has significant normative consequences. An important case is the evaluation of actions that produce immediate benefits and long-term costs, a category that includes many selfdestructive practices. Discounting induces a bias in favor of these actions, which is avoided in a time-neutral perspective. Time neutrality is therefore appealing, both as a rule of personal prudence and as a principle of social planning.

To appreciate the normative significance of temporal integration, consider an extended outcome that consists of one period of pleasure and one period of pain. Recall that integration implies the existence of a suitable monotonic transformation of instant utility (and disutility) to ratio scales (invariant up to a multiplication by a positive constant) with the same zero point. The ratio of the total durations of positive and negative periods in a mixedaffect TEO, which is observable, jointly constrains the global evaluation of the TEOs and the measures of instant utility and disutility. A globally positive evaluation of such a TEO is justified only if the ratio of the averages of (suitably transformed) utilities 
and disutilities in the two periods is more extreme than the ratios of their durations. A global positive evaluation can appear implausible when the pleasure is very brief in relation to the pain. However, an agent who is susceptible to duration neglect in the evaluation of TEOs will tend to assign positive remembered utility to outcomes that consisted of periods of intense pleasure and mild pain, with little regard for the relative durations of these experiences. We suspect, for example, that duration neglect is involved in the attitudes of addicts who do not regret their addiction. Appropriate weighting of the durations of episodes has some force as a rule of personal prudence and a principle of social planning [Broome 1991].

2. Measurement issues. There has been significant progress since Edgeworth fantasized a hedonimeter. As illustrated by the research described earlier, continuous or intermittent measurements of affect are feasible in some situations, ${ }^{6}$ where they yield utility profiles that are often quite similar for different individuals. Another promising development is the increasing sophistication of experience sampling techniques, in which individuals are prompted by a preprogrammed beeper to record various aspects of their current experience (e.g., Brandstatter [1991]). These methods are likely to be supplemented in the future by continuous or intermittent measurements of physiological indices of stress and of hedonic states.

The advantage of real-time measures of instant utility is that they avoid the biases of memory and evaluation that affect retrospective judgments of pleasure, pain, and well-being. Because they are much easier to obtain, however, retrospective measures will remain in frequent use. Their effectiveness is likely to be enhanced by procedures that reduce the impact of known biases. For example, improved estimates of the total utility of an episode could be obtained by eliciting from the respondent separate assessments of its duration and of its average instant utility. More generally, we propose that the measurement of experienced utility should be viewed as a difficult technical problem, not a hopeless quest.

3. Empirical Issues. As Figure I illustrated, the consideration of experienced utility raises a complex agenda for both theoretical

6. Several studies have shown that the requirement to report instant utility has little or no effect on subsequent global evaluations of the episode [Fredrickson and Kahneman 1993; Kahneman et al., 1993; Redelmeier and Kahneman 1996a]. 
and empirical research. Our treatment has focused on the normative question of how TEOs should be evaluated (\#3 in Figure I), and on an empirical study of the remembered and decision utility of brief aversive episodes. We found that remembered utility is determined by a Peak-End rule (\#4), in violation of temporal integration (\#5); preferences for the repetition of episodes were determined by their remembered utility (\#8). As a result, decisions failed to maximize experienced utility (\#9), although they may have maximized remembered utility (\#10). These findings should be taken as illustrative, because of the narrow range of situations in which they were obtained.

The effects of consumption on the utility of future consumption (\#2 in Figure I) play an important role in numerous economic models of individual decisions (e.g., Becker [1996], Constantinides [1990]; Gilboa [1989]). The intuitions that drive these models pertain to the dynamics of experienced utility, but the only observables that the models allow are decisions. The chain of inferences from observed decisions to experienced utility is long and, as we illustrated earlier, sometimes unreliable. We believe that experienced utility can only be understood when it is directly measured, and that a deeper understanding of experienced utility will contribute to the development of richer models of economic decisions.

In a book that has been influential outside economics, Scitovsky [1976] presented numerous ideas about experienced and predicted utility. His treatment implies that people are not generally able to solve the problem of maximizing experienced utility subject to a budget constraint (question \#9 in Figure I), at least in part because of their limited understanding and ability to predict their own enjoyment of goods and activities (\#6). He argued that American consumers tend to overinvest in comforts and to underinvest in pleasures, and suggested that members of other cultures achieve greater success in the pursuit of happiness. In the absence of measurements of experienced utility, Scitovsky could only support these strong claims by appealing to his readers' intuitions about the psychology of hedonic experience, and to their normative intuitions about the good life. At least in principle, however, his hypotheses could be studied empirically, using appropriate measures of experienced and predicted utility.

Unlike Scitovsky, Becker's analysis of individual behavior assumes that people correctly anticipate the effects of consumption on future preferences and correctly incorporate these predictions 
in their choices [Becker 1996]. The available evidence supports the more skeptical position. Studies of lay beliefs about the dynamics of taste and hedonic experience have shown these beliefs to be fragmentary and sometimes seriously inaccurate [Snell, Gibbs, and Varey 1995]. For example, the large effects of sheer familiarity in increasing liking and enjoyment are not generally known. Most people are also very surprised to learn that paraplegics are not always miserable and that lottery winners are not particularly happy [Brickman, Coates, and Janoff-Bulman 1978], reflecting a pervasive tendency to underestimate the effects of habituation.

Experimental studies of predicted utility have produced additional evidence of poor accuracy. Participants in two experiments were not able to predict how their own taste for ice cream, low-fat yogurt, or music would change over a week of repeated consumption [Kahneman and Snell 1992]. Failures of individuals to predict their own future choices were reported by Simonson [1990] and by Loewenstein and Adler [1995]. These findings are not compatible with the assumption that it is easy to anticipate future tastes and to plan consumption accordingly.

The errors of remembered utility and the consequent errors of decision that we reviewed earlier do not exhaust the difficulties that people have in evaluating extended outcomes, nor do they exhaust the ways in which decisions fail to maximize utility. Thus, many errors of predicted utility are caused by the practice of evaluating an entire extended outcome by evaluating the transition to it. For example, the mistake that most people make in predicting the well-being of paraplegics may reflect their use of the tragic event of becoming a paraplegic as a proxy in evaluating the long-term state of being a paraplegic. A similar bias is the source of many anomalies of choice. Decision makers are susceptible to framing effects and are led to inferior choices because of the pervasive tendency to evaluate outcomes as changes relative to a reference point [Tversky and Kahneman 1986, 1991]. The focus on changes sometimes induces an extreme form of myopia, where decision makers appear to evaluate a transaction by the anticipated pleasure or pain of getting or giving up a good, rather than by evaluating the state of having it [Kahneman 1994]. A detailed study of remembered utility, predicted utility, and decision utility can be expected to reveal instructive commonalities, as well as important differences.

4. Consumer Sovereignty. Some of the arguments for con- 
sumer sovereignty are called into question by the observation of decisions that systematically fall short of maximizing experienced utility. The dilemma can be illustrated by the example that introduced this paper. The amnesic patient attaches the same decision utility to the two toasters, although the experienced utility that the toasters deliver is different. The question is whether the amnesic's preferences, which clearly do not maximize experienced utility, should nevertheless be respected. This question is of interest because the difference between the patient of the story and normal people may be smaller than is commonly assumed. As illustrated by the experiments described in Section II, normal subjects also choose to expose themselves to avoidable pain because of the peculiarities of remembered utility. Do preferences that exhibit almost total duration neglect deserve respect?

The point of these observations is not to support paternalism, but to reject one of the arguments commonly raised against it. The claim that agents should be left alone because they generally know what is good for them is less secure than is generally assumed in economic discourse. A sounder case for resisting interventions in the decisions of individuals can be made on other grounds, such as the value of freedom and the high risk that coercive power will be abused. Invoking the assumption of rationality in this context merely denies the existence of a real dilemma.

\section{Conclusion}

The elimination of experienced utility from economic thought was justified by important considerations, but it was not costless. Perhaps the heaviest cost was that the exclusive concern with decision utility removed some important problems from the reach of empirical research. In particular, the proposition that people maximize utility was granted the status of a maintained hypothesis, which is used to constrain the interpretation of other facts but is not itself subject to test. Admitting experienced utility as a measure of outcomes turns utility maximization into an empirical proposition, which will probably be found to provide a good approximation to truth in many situations and to fail severely in others. The scientific merit of economic analyses that assume utility maximization will vary accordingly. Considerations of experienced utility can help identify situations in which the assumption of consumer rationality should be applied with caution, subjected to empirical test, or avoided altogether. 


\section{Appendix: A Formal Analysis and Proofs}

We denote by $[B, E[$ a time interval that contains all time points relevant to the analysis to avoid technical details. The interval is assumed bounded. For convenience of notation all time intervals are assumed left-closed and right-open in this analysis. $C$ is a set of outcomes (describing consumed commodity bundles, health states, social states, etc.) An episode is a mapping from a subinterval $[b, e$ [ to $C$, for $b \geq B$ and $e \leq E$. A TEO is a mapping from a finite disjoint union of subintervals of $[B, E$ [ to $C$.

The set of levels of instant utilities is denoted by $X . X$ is an interval that contains 0 ; it may be bounded or unbounded. To each TEO we assign a dated utility profile, i.e., a function defined on the same domain as the TEO, but assigning to each time point of the domain a level of instant utility instead of an outcome. The instant utility at a time point depends on the outcome associated with that time point, but also on outcomes associated with other time points.

Our analysis has been based on the assumption of no discounting. Hence a dated utility profile defined on some interval $[b, e[$ is equivalent to a dated utility profile on an interval $[b+t$, $e+t$ [ with corresponding instant utilities, for any $t$. We only need to know the time duration $e-b$ of the interval, and the instant utility profile over that interval, and we need not know the value of $b$ or $e$. Therefore, we assign to each dated utility profile $f^{*}$, defined on a time interval $[b, e[$, a mapping $f$ with domain $[0, e-b$, such that $f(t)=f^{*}(b+t)$ for all $t$. $f$ is called the undated utility profile, or utility profile for short. Loosely speaking, we set the clock at zero at the beginning of each utility profile; i.e., we use stopwatch time instead of calendar time. The definition reflects the assumption that an hour of some specific level of instant utility yields the same amount of total utility, independent of when it happens in history.

A utility profile, or profile for short, is a bounded function from an interval [0, $d$ [ to $X$, where $0<d<M$ with $M$ the maximal duration that is conceivable; i.e., $M=E-B .{ }^{7}$ The utility profile is complete if its domain is [0,M[. For two profiles $f, g$ defined on $[0, a$ [ and [0,b [, respectively, with $a+b \leq M$, the concatenation $f \& g$ is defined on $[0, a+b$ [ and coincides with $f$ on [0, $a$ [ and with $g$ on $[a, a+b[$ (after appropriate translation of the arguments).

7. We make the usual measurability assumptions; i.e., utility profiles are "Lesbesgue-measurable" [Halmos 1950]. 
Next we define utility profiles for TEOs. A TEO can be considered a concatenation of episodes, and we can consider the utility profiles belonging to the separate episodes of a TEO. The utility profile belonging to a TEO is defined as the concatenation of the utility profiles of the episodes that the TEO consists of. Its duration is therefore the total duration of the TEO, i.e., the sum of the durations of the separate episodes. By using the concatenation of the separate utility profiles, we drop the information regarding the time spans between the separate episodes. That is, again, a reflection of our assumption of zero discounting. The assumption would be eminently unreasonable if utility profiles would assign outcomes to time points instead of instant utilities, and illustrates the essential role of instant utility in our analysis.

To each episode and TEO, a utility profile is assigned through the above process, and the total utility of an episode or TEO is simply the total utility of the belonging utility profile. The rest of the analysis will only concern utility profiles.

$\succcurlyeq$ is the weak ordering of profiles represented by total utility; i.e., $f \geqslant g$ if and only if the total utility of $f$ is at least as high as that of $g$. We assume at first that total utility is measured at an ordinal level, meaning that $\succcurlyeq$ comprises all relevant information. Therefore, the first part of our analysis is in terms of $\succcurlyeq$. The restriction of $\succcurlyeq$ to complete profiles is denoted by $\succcurlyeq_{c}$.

We next turn to Axiom 3, monotonicity of $\succcurlyeq$ with respect to total utility. We first give a formal statement of the axiom.

Axiom A.1 [monotonicity in total utility, formal statement of Axiom 3]. If $f \succcurlyeq g$, then $f \& h \succcurlyeq g \& h$ and $h \& f \succcurlyeq h \& g$ whenever these concatenations are defined. Strict preference in the premise implies strict preference in the conclusion.

We next demonstrate how versions of separability of $\geqslant$ follow from monotonicity with respect to total utility. We say that $\succcurlyeq_{c}$ satisfies separability if

$$
f \succcurlyeq_{c} g \text { implies } f^{\prime} \succcurlyeq_{c} g^{\prime}
$$

whenever, for a partition $\{I, R\}$ of $\left[0, M\left[, f=g\right.\right.$ and $f^{\prime}=g^{\prime}$ on $I$ and $f=f^{\prime}, g=g^{\prime}$ on $R$. $\succcurlyeq_{c}$ satisfies left separability if (A.2) holds only for the special case in which $I=[0, e$ [ for some $e$, and $\succcurlyeq_{c}$ satisfies right separability if (A.2) holds only for the special case in which $I=[b, M$ [ for some $b$. 
Lemma A.1. Under Axiom 1 (neutral concatenation), monotonicity in total utility implies left- and right-separability for $\succcurlyeq_{c}$.

Proof. For left-separability, let $f, f^{\prime}, g, g^{\prime}$ be as above, and assume that $I=\left[0, e\left[\right.\right.$. We can write $f=c \& x, g=c \& y, f^{\prime}=c^{\prime} \& x$, $g^{\prime}=c^{\prime} \& y$ (define $c$ as the restriction of $f(=g)$ on $[0, e[, x$ as the restriction of $f\left(=f^{\prime}\right)$ on $[e, M[$, "shifted leftwards," etc.). By monotonicity in total utility, we have

$\begin{array}{lll}x \succ_{y \text { implies }} & c \& x \succ_{c \& y} \text { and also } & c^{\prime} \& x \succ_{c^{\prime} \& y .} \\ x \prec y \text { implies } & c \& x \prec c \& y \text { and also } & c^{\prime} \& x \prec c^{\prime} \& y . \\ x \sim y \text { implies } & c \& x \sim c \& y \text { and also } & c^{\prime} \& x \sim c^{\prime} \& y \\ & & \text { (apply the } \\ & & \text { condition both } \\ & & \text { with } \succcurlyeq \text { and } \preccurlyeq) .\end{array}$

That is, in each case the preference between $f$ and $g$ agrees with that between $f^{\prime}$ and $g^{\prime}$. This is what left-separability requires.

Right-separability is derived similarly, considering $f^{\prime}=x \& c$, $g=y \& c, f=x \& c, g^{\prime}=y \& c^{\prime}$.

Our technical condition concerns continuity with respect to the supremum norm. A sequence of profiles $f^{j}$ converges to a profile $f$ (in supremum norm) if these profiles are all of the same duration, and for each positive $\varepsilon$ there exists a natural number $N$ such that $\left[f^{j}(t)-f(t) \mid \leq \varepsilon\right.$ for all $j \geq N$ and $t$ in the domain. Supnorm-continuity means that, for every sequence $f^{j}$ converging to $f$ in supremum norm, and every $g \succ_{f}$, there exists a natural number $N$ such that $g \succ f^{j}$ for all $j \geq N$, and the same holds with $\prec$ instead of $\succ$ everywhere. We now state the main theorem formally.

Theorem A.1 [formal version of Theorem 1]. Let $M>0$. For a binary relation $\succcurlyeq$ defined over the (utility) profiles, i.e., all measurable bounded mappings from subintervals [0,e[ of $[0, M$ [ to an interval $X$ containing 0 , the following two statements are equivalent.

(i) There exists a nondecreasing continuous ("value") function $v: X \rightarrow I R$, assigning value 0 to 0 , such that profiles are ordered according to the integral of the value of instant utility over time.

(ii) $\succcurlyeq$ is a supnorm-continuous weak order, Axiom 1 (neutral concatenation), Axiom 2 (monotonicity in instant utility), and Axiom 3 (monotonocity in total utility, i.e., Axiom A.1) hold.

Furthermore, $v$ in (i) is a ratio scale. 
Proof. Since the implication (i) $\Rightarrow$ (ii) is easily established, we assume (ii) and derive (i), as well as the uniqueness result. As the theorem is trivial if all levels of instant utility are equivalent (then all profiles are equivalent by Axiom 2, and $v=0$ ), we assume that there are two levels of instant utility that are not equivalent. Because of neutral concatenation, we will mostly restrict attention to the weak ordering $\succcurlyeq_{c}$ of the complete profiles defined on all of [0,M[. By Lemma A.1, monotonicity in total utility implies left- and right-separability of $\succcurlyeq_{c}$.

For some $n \geqslant 3$, we now partition the interval [0, $M$ [ into $n$ subintervals $\left[m_{0}, m_{1}\left[, \ldots,\left[m_{n-1}, m_{n}\right.\right.\right.$ [ of equal length $M / n\left(m_{0}=0\right.$, $\left.m_{n}=M\right)$. By $\left(x_{1}, \ldots, x_{n}\right)$ we denote the complete profile that assigns $x_{j}$ to all time points in the $j$ th interval. This set of complete profiles, denoted by $F^{n}$, is isomorphic to $X^{n}$, and we now study the restriction $\succcurlyeq_{r}$ of $\succcurlyeq_{c}$ to $F^{n}$. We first show that any interval $\left[m_{j-1}, m_{j}\right.$ [ is essential with respect to $\succcurlyeq_{r}$, i.e., profiles from $F^{n}$ that agree outside of the interval need not be equivalent.

Assume that the level of instant utility $x$ is strictly preferred to $y$; i.e., the complete profile that is constant $x$ is strictly preferred to the complete profile that is constant $y$. Assume, for contradiction, that $x\left[m_{j-1}, m_{j}\left[\sim y\left[m_{j-1}, m_{j}\right.\right.\right.$, where we follow the convention of using the set $\left[m_{j-1}, m_{j}\right.$ [ to denote also the indicator function that is 1 on $\left[m_{j-1}, m_{j}\right.$ [ and 0 on the rest of $[0, M[$. By Axiom 1, $x\left[m_{i-1}, m_{i}\left[\sim y\left[m_{i-1}, m_{i}\right.\right.\right.$ [ also holds for all $i$ different than $j$. Then, by left- and right-separability, $y\left[0, M\left[\sim x\left[0, m_{1}\left[+y\left[m_{1}\right.\right.\right.\right.\right.$, $M\left[\sim x\left[0, m_{2}\left[+y\left[m_{2}, M\left[\sim \ldots \sim x\left[0, m_{n-1}\left[+y\left[m_{n-1}, M[\sim x[0, M[\right.\right.\right.\right.\right.\right.\right.\right.$, contradicting our assumption that $y[0, M[\prec x[0, M[$. So every interval $\left[m_{j-1}, m_{j}\right.$ [ is essential with respect to $\succcurlyeq_{r}$.

Because all intervals are essential and $n \geq 3$, and $\succcurlyeq_{r}$ is a continuous weak order that satisfies left- and right-separability, it satisfies full-force separability by Gorman [1968], and there exist functions $V_{1}, \ldots, V_{n}$ such that $V_{1}\left(x_{1}\right)+\ldots+V_{n}\left(x_{n}\right)$ represents $\succcurlyeq_{r}$ of $F^{n}$. We may, and will, assume that $V_{j}(0)=0$ for all $j$. Because Axiom $1,(x, 0, \ldots, 0) \sim(0, x, 0, \ldots, 0) \sim \ldots \sim(0, \ldots, 0, x)$, and this implies that $V_{1}(x)=\ldots=V_{n}(x)$ for all $x$. We define $v(x)=V_{1}(x) /$ $\left(m_{1}-m_{0}\right)$. We can then conclude that the integral of $v$ represents preferences over $F^{n}$. By Axiom 2, $v$ is nondecreasing. As the above analysis holds true for all $n$ and thus for a dense subset of the complete profiles, supnorm-continuity implies that $\succcurlyeq_{c}$ is represented by the integral of $v$ on the whole set of complete profiles. By zero-concatenation it now follows that the representation extends to all profiles.

Gorman [1968] also demonstrated that the $V_{j}$ functions de- 
rived above are unique up to location and a common scale. This, their unique relatedness to $v$, and $v(0)=0$, imply that $v$ is a ratio scale.

Proof of Theorem 2. We assume the same technical conditions ( $M$, domain, and supnorm-continuity) as in Theorem A.1. The necessity of Axiom 4 for linearity of the value function is obvious-hence we assume the axiom and derive the representation. Note that we do not have Axiom 3 (A.1) available. Therefore, we must derive it in some way. A step profile is a finite concatenation of constant profiles; i.e., it is finite-valued, and it is constant over intervals.

\section{Lemma A.2. Axiom 4 implies Axiom 3 (A.1) for step profiles.}

Proof. Assume that, for step profiles $x, y, c$, we have $x \geqslant y$, and that $c \& x$ and $c \& y$ are defined. First, assume that $c$ is constant. $c^{\prime}$ denotes the neutral profile of the same duration as $c$. By neutral concatenation, we get $c^{\prime} \& x \sim x \geqslant y \sim c^{\prime} \& y$. By Axiom 4 we can add up the instant utility of $c$ over the duration of $c$ and get $c \& x \geqslant \mathrm{e}$ $c \& y$. We can repeat this for other constant profiles $c_{1}, \ldots, c_{n}$, to get $c_{n} \& \ldots \& c_{1} \& c \& x \geqslant c_{n} \& \ldots \& c_{1} \& c \& y$. Every step-profile $c^{\prime \prime}$ can be written as a concatenation $c_{n} \& \ldots \& c_{1} \& c$, and hence we get $c^{\prime \prime} \& x \geqslant c^{\prime \prime} \& y$ for all step profiles $c^{\prime \prime}$. Similarly, $x \& c^{\prime \prime} \geqslant y \& c^{\prime \prime}$ follows. The same reasoning also applies to strict instead of weak preferences.

In the proof of Theorem 1/A.1, we only needed Axiom 3 (A.1) and its implications (e.g. left- and right-separability), and in fact all conditions except weak ordering and supnorm-continuity, for step profiles. Hence we can invoke the representation of the theorem. Partition $[0, M[$ into $[0, M / 2[$ and $[M / 2, M[$. The notation $\left(x_{1}, x_{2}\right)$ designates here the profile that is $x_{1}$ on the first interval and $x_{2}$ on the second. Now $(x, 0) \sim(x, 0)$-hence, by Axiom $4,(x, \varepsilon)$ $\sim(x+\varepsilon, 0)$. This implies that $v(x+\varepsilon)-v(x)=v(\varepsilon)$ for all $x$ and $\varepsilon$; i.e., $v$ satisfies the Cauchy equation. For the nondecreasing $v$ that implies that $v$ is linear.

QED

All the results above have assumed total utility only at the ordinal level, and have characterized total utility as an increasing transform of an integral (because it induces the same ordering over profiles as the integral). We next consider the case in which total utility is measurable at the cardinal level. That can be tested by the following axiom. 
Axıом A.2 [cardinal total utility]. The total utility of two concatenated profiles is the sum of their separate total utilities.

In the presence of Axiom A.2, Theorems 1 and 2 can be strengthened to provide total utility as an integral of the value of instant utility and the integral of instant utility, respectively. We assume in the theorems below the same technical conditions $(M$, domain, weak ordering, supnorm-continuity) as in Theorem A.1.

Theorem A.2. Axioms 1, 2, and A.2 hold if and only if total utility is an integral of the value of instant utility.

Proof. Necessity of the axioms is obvious - hence we assume the axiom and derive the representation. Axiom A.2 implies Axiom 3, and hence we obtain the integral representation of Theorem A.1. Note that the integral of the value function does satisfy Axiom A.2.

Let $\left(x_{1}, x_{2}\right)$ be the complete profile that is $x_{1}$ on $\left[0, M / 2\left[\right.\right.$ and $x_{2}$ on $[M / 2, M[$. Both total utility and the integral of the value function provide additively decomposable representations for $\succcurlyeq$, that both assign 0 to the neutral profile. By standard uniqueness results on additive conjoint measurement (continuity of total utility need not be presupposed here but is implied, see Wakker, [1988]), total utility must be a positive scalar times the integral. We can divide the value function by that positive scalar.

Corollary A.1. Under the technical conditions of Theorem A.3, Axioms 1, 2, 4, and A.2 hold if and only if total utility is the integral of instant utility.

Woodrow Wilson School of Public Affairs, Princeton University Medical Decision Making Unit, Leiden University, The Netherlands Anderson School of Business, University of California, Los Angeles

\section{REFERENCES}

Becker, G. S., Accounting for Tastes (Cambridge, MA: Harvard University Press, 1996).

Bentham, J., An Introduction to the Principle of Morals and Legislations (1789); reprinted (Oxford, UK: Blackwell, 1948).

Brandstatter, H., "Emotions in Everyday Life Situations: Time Sampling of Subjective Experience," in F. Strack, M. Argyle, and N. Schwarz, eds., Subjective Well-Being: An Interdisciplinary Perspective (Oxford: Pergamon, 1991), pp. 173-92.

Brickman, P., D. Coates, and R. Janoff-Bulman, "Lottery Winners and Accident Victims: Is Happiness Relative?" Journal of Personality and Social Psychology, XXXVII (1978), 917-27.

Broome, J., Weighing Goods (Oxford, UK: Blackwell, 1991).

Cabanac, M., "Physiological Role of Pleasure," Science, CLXXIII (1971), 1103-07. 
Clark, A. E., and A. J. Oswald, "Unhappiness and Unemployment," Economic Journal, CIV (1994), 648-659.

Constantinides, G. M., "Habit Formation: A Resolution of the Equity Premium Puzzle," Journal of Political Economy, XCVIII (1990), 519-43.

Dasgupta, P., An Inquiry into Well-Being and Destitution (Oxford: Clarendon, 1993).

Edgeworth, F. Y., Mathematical Psychics: An Essay on the Application of Mathematics to the Moral Sciences (1881); reprinted (New York: M. Kelly, 1967).

Frank, R. H., Passions within Reason: The Strategic Value of the Emotions (New York: Norton, 1988).

Fredrickson, B. L., and D. Kahneman, "Duration Neglect in Retrospective Evaluations of Affective Episodes," Journal of Personality and Social Psychology, LXV (1993), 45-55.

Gilboa, I., "Expectations and Variations in Multi-Period Decisions," Econometrica, LVII (1989), 1153-69.

Gorman, W. M., "The Structure of Utility Functions," Review of Economic Studies, XXXV (1968), 367-90.

Gottman, J. M., and R. W. Levenson, "A Valid Procedure for Obtaining Self-Report of Affect in Marital Interaction," Journal of Consulting and Clinical Psychology, LIII (1985), 51-160.

Halmos, P. R., Measure Theory (New York: Van Nostrand, 1950).

Kahneman, D., "New Challenges to the Rationality Assumption," Journal of Institutional and Theoretical Economics, CL (1994), 18-36.

Kahneman, D., B. L. Fredrickson, C. A. Schreiber, and D. A. Redelmeier, "When More Pain Is Preferred to Less: Adding a Better End," Psychological Science, IV (1993), 401-05.

Kahneman, D. and J.S. Snell, "Predicting a Changing Taste: Do People Know What They Will Like?" Journal of Behavioral Decision Making, V (1992), $187-200$.

Kahneman, D., and C. Varey, "Notes on the Psychology of Utility," in J. Elster and J. E. Roemer, eds., Interpersonal Comparisons of Well-Being: Studies in Rationality and Social Change (New York: Cambridge, 1991), pp. 127-63.

Kapteyn, A., "The Measurement of Household Cost Functions: Revealed Preference versus Subjective Measures," Journal of Population Economics, VII (1994), 333-50.

Loewenstein, G., "The Fall and Rise of Psychological Explanations in the Economics of Intertemporal Choice," in G. Loewenstein and J. Elster, eds., Choice Over Time (New York: Russell Sage Foundation, 1992), pp. 3-34.

Loewenstein, G., and D. Adler, "A Bias in the Prediction of Tastes," Economic Journal, CV (1995), 929-37.

Mowrer, O. H., and L. N. Solomon, "Contiguity vs. Drive-Reduction in Conditioned Fear: The Proximity and Abruptness of Drive-Reduction," American Journal of Psychology, LXVII (1954), 15-25.

Nussbaum, M. C., and A. Sen, The Quality of Life (Oxford, UK: Clarendon, 1993).

Redelmeier, D., and D. Kahneman, "Patients' Memories of Painful Medical Treatments: Real-Time and Retrospective Evaluations of Two Minimally Invasive Procedures," Pain, CXVI (1996a), 3-8.

Redelmeier, D., and D. Kahneman, "Improving the Memory of a Colonoscopy," Working Paper, 1996b.

Schelling, T. C., Choice and Consequence: Perspectives of an Errant Economist (Cambridge, MA: Harvard University Press, 1984).

Scitovsky, T., The Joyless Economy (New York: Oxford University Press, 1976).

Schreiber, C. A., and D. Kahneman, "Beyond the Peak and End Hypothesis: Exploring the Relation between Real-Time Displeasure and Retrospective Evaluation," Working Paper, Princeton University, 1996.

Sen, A. K., "Welfare, Preference and Freedom," Journal of Econometrics, L (1991), 15-29.

Simonson, I., "The Effect of Purchase Quantity and Timing on Variety-Seeking Behavior," Journal of Marketing Research, XXVII (1990), 150-62.

Snell, J., B. J. Gibbs, and C. Varey, "Intuitive Hedonics: Consumer Beliefs about the Dynamics of Liking," Journal of Consumer Psychology, IV (1995), 33-60.

Stevens, S. S., Psychophysics: Introduction to its Perceptual, Neural and Social Prospects (New York: Wiley, 1975). 
Stigler, G. J., “The Development of Utility Theory: I; II," Journal of Political Economy, LVIII (1950), 307-27; 373-96.

Stigler, G. J., and G. S. Becker, "De Gustibus non Est Disputandum," American Economic Review, LXVII (1977), 76-90.

Tinbergen, J., "On the Measurement of Welfare," Journal of Econometrics, L (1991), 7-13.

Tversky, A., and D. Kahneman, "Rational Choice and the Framing of Decisions," Journal of Business, CIX (1986), S251-78.

Tversky, A., and D. Kahneman, "Reference Theory of Choice and Exchange," Quarterly Journal of Economics, CVI (1991), 1039-61.

Van Praag, B. M. S., "Ordinal and Cardinal Utility: An Integration of the Two Dimensions of the Welfare Concept," Journal of Econometrics, L (1990), 69-89.

Varey, C., and D. Kahneman, "Experiences Extended across Time: Evaluation of Moments and Episodes," Journal of Behavioral Decision Making, V (1992), 169-86.

Wakker, P. P., "The Algebraic versus the Topological Approach to Additive Representations," Journal of Mathematical Psychology, XXII (1988), 421-35.

Wegener, B., ed., Social Attitudes and Psychophysical Measurements (Hillsdale, NJ: Lawrence-Erlbaum, 1982). 